\title{
Análise do Tratamento Químico em Carvão Ativado para Remoção do Corante Azul 5g
}

\author{
Noemi H. de Melo, Maria E. O. Ferreira, Nathan F. F. Potenciano, \\ Paulo R. Martins \& Indianara C. Ostroski
}

A maioria dos estudos em adsorção são realizados em sistemas batelada, que são limitados a escala laboratorial e tem menor aplicação nos processos industriais. Os leitos fixos são particularmente interessantes devido à sua fácil operação e possibilidade de regeneração sem que o leito seja desmontado. Assim, o presente trabalho teve por objetivo estudar o equilíbrio de adsorção do corante reativo azul 5G utilizando carvão ativado de osso de boi bruto e tratado com $\mathrm{NaOH}$, em sistemas de leito-fixo. Testes dinâmicos foram realizados com o fluxo ascendente em coluna, a $30{ }^{\circ} \mathrm{C}$ e diâmetro médio de partícula de $0,0765 \mathrm{~mm}$. A vazão ótima de trabalho encontrada para ambos os adsorventes foi de $2 \mathrm{~mL}$ min- 1 . As isotermas foram obtidas pelo balanço de massa nas curvas de ruptura, e foram ajustadas aos modelos de Langmuir e Freundlich. O carvão ativado de osso de boi demonstrou ter potencial para ser usado como um adsorvente alternativo na remoção do corante azul 5G presente em efluentes têxteis. E na adsorção também foi verificado que a funcionalização da superfície química do carvão não foi significativa no processo.

\section{Palavras-chave: Adsorção; Leito Fixo; Funcionalização.}

The majority of adsorption studies have been carried out in batch models, which are limited to a laboratory scale and has less application in industrial processes. The fixed beds are particularly interesting due to their easy operation and possibility of regeneration without the bed being dismantled. Thus, the present work had the objective of studying the adsorption equilibrium of reactive blue $5 \mathrm{G}$ dye by using raw bone char and its $\mathrm{NaOH}$ treated form in bed-fixed system. Dynamic tests were performed with the column flow rising at $30{ }^{\circ} \mathrm{C}$ and mean particle diameter of $0.0765 \mathrm{~mm}$. The optimum flow-rate found for both adsorbents was $2 \mathrm{~mL}$ min-1. The dynamic isotherms were obtained by the mass balance in the rupture curves, and were adjusted to the Langmuir and Freundlich models. Bone char demonstrated potential to be used as an alternative adsorbent in the removal of blue $5 \mathrm{G}$ dye present in textile effluents. And in the adsorption it was also verified that the functionalization of the chemical surface of the adsorbent was not significant in the process.

Keywords: Adsorption; Fixed Bed; Functionalization. 


\section{Introdução}

A indústria têxtil desempenha um papel importante na economia brasileira. Segundo a Associação Brasileira da Indústria Têxtil e Confecção (Abit), em 2016 a produção média têxtil foi de 1,7 milhão de toneladas, colocando o país como o quinto maior produtor deste setor do mundo ${ }^{1}$. Os resultados promissores fazem com que haja cada vez mais investimentos no setor têxtil, com o objetivo de atingir um desenvolvimento e um faturamento adequados para competir no mercado externo.

Entretanto, do ponto de vista ambiental, o setor é considerado potencialmente poluidor. Os efluentes gerados nos processos da indústria têxtil apresentam uma carga de cor muito elevada devido ao uso de corantes e sua baixa fixação nas fibras durante processo de tingimento. Além de problemas estéticos, a alteração na cor decorre na redução da penetração da luz e consequentemente comprometimento da vida aquática2. Por isso, tais efluentes devem ser tratados conforme procedimentos estabelecidos por normas e leis antes do descarte nos corpos hídricos ${ }^{3}$.

Os tratamentos usualmente aplicados na remoção de corantes sintéticos em efluentes envolvem o uso de tecnologias tais como separação por membranas, oxidação eletroquímica, foto-oxidação, adsorção, coagulação e tratamentos biológicos envolvendo enzimas ${ }^{4,5}$. A adsorção se destaca dentre estas técnicas devido a sua simplicidade e elevada eficiência ${ }^{6,7}$.

A adsorção é descrita por Ruthven (1984)8 como uma operação de transferência de massa, na qual se explora a capacidade de certos sólidos (adsorventes) em concentrar na sua superfície determinadas substâncias existentes em fluidos líquidos ou gasosos, possibilitando a separação dos componentes desses fluidos.

Diversos adsorventes têm sido testados com o objetivo de reduzir a concentração de corantes em efluentes aquosos ${ }^{9-16}$. Dentre esses materiais, os carvões ativados têm se mostrado muito eficientes, pois apresentam elevada capacidade de adsorção para vários compostos orgânicos. Isso se dá, principalmente porque possuem uma estrutura de poros altamente desenvolvida, elevada área superficial, baixa densidade e alta resistência química. A princípio, qualquer material com alto teor de carbono, denominado agente precursor, pode ser transformado em carvão ativado. É o caso do carvão ativado de osso de boi, obtido a partir da pirólise de resíduos de osso bovino.

O carvão ativado de osso de boi é um adsorvente alternativo, composto principalmente de hidroxiapatita (70$76 \%$ ), carbonato de cálcio (7-9\%) e uma fase de carbono amorfo $(9-11 \%)^{17-19}$. Apresenta propriedades atraentes para tratamento e purificação de água e vários autores relataram sua aplicação geral na remoção de compostos orgânicos e inorgânicos ${ }^{17,19-23}$. No entanto, são poucos os estudos que relataram a remoção de corantes em efluentes têxteis usando o adsorvente em questão ${ }^{24-27}$.

Os estudos em adsorção podem ser realizados tanto em sistemas batelada quanto em colunas de leito fixo. Este último é interessante devido à facilidade de operação e à possibilidade de regeneração sem que o leito seja desmontado $^{28}$. Desta forma, o objetivo deste trabalho é realizar um estudo do mecanismo de adsorção do corante reativo Azul 5G em carvão ativado de osso de boi puro e tratado quimicamente com solução de hidróxido de sódio $(\mathrm{NaOH})$, utilizando sistema de leito fixo. O corante reativo azul $5 \mathrm{G}$ é conhecido por ser muito utilizado no tingimento de algodão e em lavanderias industriais, no tingimento do jeans.

\section{Materiais e Métodos}

\section{ADSORVENTE E ADSORVATO}

Para a realização dos experimentos utilizou-se efluente sintético preparado com o corante reativo Azul 5G, o qual é produzido pela Texpal Indústria Química S/A. O corante foi utilizado sem tratamento prévio e as soluções foram feitas utilizando-se água destilada. Para a determinação da concentração de corante nas amostras foi utilizado um espectrofotômetro e comprimento de onda igual a $600 \mathrm{~nm}$.

O adsorvente utilizado nos ensaios para a remoção do corante foi o carvão ativado de osso de boi, doado pela Bonechar Carvão do Brasil Ltda. A determinação do diâmetro de partícula médio do adsorvente foi feita por 
peneiramento. Neste trabalho utilizou-se o diâmetro de partícula igual a de $0,0765 \mathrm{~mm}$.

\section{TRATAMENTO DO CARVÃO}

Duas amostras diferentes de carvão ativado foram consideradas nesse trabalho: o carvão de osso de boi puro (COB) e o carvão de osso de boi funcionalizado quimicamente com solução de hidróxido de sódio (COT). Para isso, preparou-se $100 \mathrm{~mL}$ de uma solução de $\mathrm{NaOH}_{2}$ mol L ${ }^{-1}$, à qual foram adicionados $20 \mathrm{~g}$ de carvão ativado de osso de boi. Posteriormente, a solução foi submetida a um processo de agitação numa incubadora shaker da marca Ethik modelo 430/RBD, por um período de $48 \mathrm{~h}$ e a temperatura constante de $30{ }^{\circ} \mathrm{C}$. Finalizado tal procedimento, o carvão foi, então, filtrado e, em seguida, seco em estufa durante $48 \mathrm{~h}$. As caracterizações físicas e químicas das amostras do carvão bruto e tratado foram realizadas e os resultados podem ser consultados em Winter et al. $(2016)^{29}$.

\section{PREPARAÇÃO E OPERAÇÃO DAS COLUNAS DE LEITO FIXO}

Para os ensaios em coluna com leito fixo, foi utilizado um módulo que consiste em dois reservatórios para soluções de corante (alimentação) e um para água destilada, usada na lavagem do leito e das mangueiras, e no ajuste de vazão. Há também duas bombas peristálticas (ColeParmer) que alimentam as colunas, cujo diâmetro interno é de 0,9 $\mathrm{cm}$, além de um banho termostático (Solab) que alimenta as camisas das colunas, mantendo a temperatura constante. $\mathrm{O}$ esquema da unidade de adsorção é mostrado na Figura 1.

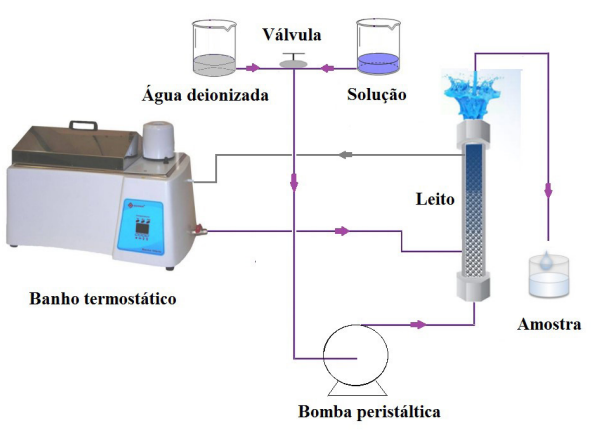

Figura 1. Unidade experimental.
Montou-se o leito de forma que um terço da coluna fosse preenchida com esferas de vidro de diâmetro aproximado de 3,94 nm. Isto foi feito para sustento do leito e para criar uma zona de normalização do fluxo em sua entrada. Logo após, colocou-se um pequeno pedaço de algodão em cima das esferas, com o objetivo de impedir que o carvão ativado penetrasse entre elas. Adicionou-se $1 \mathrm{~g}$ de adsorvente por cima do algodão, o qual foi coberto com outro pedaço de algodão, e completou-se a coluna com esferas de vidro. A altura do carvão na coluna foi de $2,5 \mathrm{~cm}$ e a temperatura do banho foi ajustada em $30^{\circ} \mathrm{C}$.

\section{ESTUDO DA VAZÃO ÓTIMA DE OPERAÇÃO}

Para a obtenção da vazão ótima do corante Azul $5 \mathrm{G}$, avaliou-se vazões as de 2, 4, 6, 8, 10 e $12 \mathrm{~mL} \mathrm{~min}^{-1}$, utilizando-se concentrações fixas de alimentação iguais a 55 e $173 \mathrm{mg} \mathrm{L}^{-1}$ para o COB e COT, respectivamente. Assim, foram construídas curvas de ruptura, a partir das quais foram calculados os parâmetros de transferência de $\operatorname{massa}^{30}$.

No processo de adsorção, os parâmetros de transferência de massa são extremamente importantes, uma vez que por meio deles é possível verificar se o processo é favorável ou não. Um dos principais parâmetros é o comprimento da zona de transferência de massa $(Z T M)$, que pode ser calculado por meio da Eq. $(1)^{30}$ :

$$
\mathrm{ZTM}=(1-\tau) \mathrm{H}_{\mathrm{t}}
$$

em que $H_{t}$ é a altura total do leito e $\tau=t_{u} / t_{t}$, sendo este parâmetro considerado como a fração do comprimento útil da coluna até o ponto de ruptura (PR). $\mathrm{O}$ tempo equivalente à capacidade útil da coluna $\left(t_{u}\right)$ e o tempo equivalente à capacidade total do leito $\left(t_{t}\right)$, podem ser obtidos, respectivamente, pelas Equações (2) e (3): 


$$
\begin{aligned}
& t_{u}=\int_{0}^{t_{b}}\left(1-\frac{C_{\text {out }}}{C_{0}}\right) d t \\
& t_{t}=\int_{0}^{\infty}\left(1-\frac{C_{\text {out }}}{C_{0}}\right) d t
\end{aligned}
$$

em que o limite de integração $t_{b}$, na Eq. 2, é o tempo de ruptura, que corresponde ao tempo em que se atinge o ponto de ruptura, isto é, $5 \%$ da concentração inicial do soluto $\left(C_{b}=0,05 C_{0}\right)^{31}$. Os termos Co e Cout correspondem as concentrações de alimentação e da saída da coluna em $\mathrm{mg} \mathrm{L}{ }^{-1}$, respectivamente.

Outro parâmetro pertinente a ser analisado é o tempo de resistência médio $(t \overline{)}$ :

$$
\bar{t}=\int_{0}^{\infty} t E(t) d t
$$

em que $E(t)$ é a distribuição do tempo de resistência do fluido, que é calculado pela derivação da curva de ruptura do processo de adsorção dada por $F(t)$ nas Equações (5) e (6):

$$
\begin{aligned}
& E=\frac{d F}{d t} \\
& F=\frac{C_{\text {out }}}{C_{0}}
\end{aligned}
$$

A relação entre a condição operacional ideal e a condição real é dada pela razão operacional $\left(\mathrm{R}_{0}\right)$. Valores mais próximos de zero indicam que a condição operacional se encontra mais próxima da ideal. Esta análise fornece as condições mínimas de resistências difusionais. A razão operacional é dada pela Eq. (7) como mostrada em Pereira et al. (2006) ${ }^{32}$ :

$$
R_{0}=\left|\frac{\bar{t}-t_{u}}{t_{u}}\right|
$$

Posteriormente, também analisou-se a capacidade dinâmica da coluna, que é a quantidade de corante retida no leito até o ponto de ruptura. AEq. (8) fornece este parâmetro ${ }^{33}$ :

$$
U_{i}^{t_{b}}=\frac{C_{0} \cdot Q}{1000 \cdot m_{s}} t_{u}
$$

em que $Q$ é a vazão volumétrica da solução de corante em $\mathrm{mL} \min ^{-1}$ e $M_{S}$ é a massa de adsorvente em gramas.

\section{Isotermas de adsorção}

Com a vazão ótima de trabalho determinada em cada sistema, obteve-se os dados de equilíbrio para a adsorção do corante em COB e COT. A unidade experimental, assim como o procedimento de montagem e operação das colunas foram os mesmos para o ajuste da vazão, conforme apresentados na seção anterior.

Para este estudo, variou-se a concentração de alimentação de $10 \mathrm{mg} \mathrm{L}^{-1}$ à $275 \mathrm{mg} \mathrm{L}^{-1}$, e a temperatura foi fixada a $30^{\circ} \mathrm{C}$. Da mesma forma como na determinação da vazão ótima, os ensaios para construção das curvas de ruptura foram realizados até a completa saturação do leito. Por meio da Eq. (9), calculou-se a quantidade de corante adsorvido $\left(q_{e}\right)$ ao longo da coluna de leito fixo pelo carvão ativado em cada uma das concentrações avaliadas:

$$
q_{e}=\frac{C_{0} Q}{1000 \cdot m_{s}} \int_{0}^{t}\left(1-\frac{C_{\text {out }}}{C_{0}}\right) d t
$$


A partir dos dados de $q_{e}$ calculados, em função das concentrações de alimentação $\left(C_{\theta}\right)$ utilizadas, os mesmos foram ajustados pelos modelos de Freundlich e Langmuir. O modelo de Langmuir é representado pela Eq. (10), em que $K_{L}$ e $q_{\max }$ são as constantes relacionadas respectivamente com a energia livre de adsorção e a máxima adsorção possível:

$$
q_{e}=\frac{q_{\max } \cdot K_{L} \cdot C_{e}}{1+K_{L} \cdot C_{e}}
$$

O parâmetro $R_{L}$, permite prever a forma da isoterma de adsorção, indicando se a adsorção é favorável ou desfavorável e pode ser calculado pela Eq. (11):

$$
R_{L}=\frac{1}{1+K_{L} C_{e}}
$$

A isoterma é desfavorável quando $R_{L}>1$, linear quando $R L=1$, favorável se $R_{L}<1$ e irreversível se $R_{L}=0$.

$\mathrm{O}$ modelo da isoterma de Freundlich é descrito pela Eq. (12), em que KF e n são as constantes e se relacionam com a distribuição dos sítios ativos e a capacidade de adsorção do adsorvente:

$$
q_{e}=K_{F} \cdot C_{e}^{\frac{1}{n}}
$$

\section{Resultados e Discussão}

\section{ESTUDO DO EFEITO DA VAZÃO VOLUMÉTRICA}

As curvas de ruptura obtidas com a variação de vazão para ambos os adsorventes são apresentadas na Figura 2. A saturação para as vazões de 2, 4, 6, 8, 10 e $12 \mathrm{~mL} \mathrm{~min}^{-1}$ ocorreu em 740, 470, 390, 210, 105 e 50 minutos para o COB, e em 360, 180, 150, 120, 105 e 90 min para o COT, respectivamente. Desta forma, observa-se que o aumento da vazão de alimentação conduziu a uma saturação mais rápida dos adsorventes, comportamento este que já era esperado. Nas Figuras $2 \mathrm{a}$ e $2 \mathrm{~b}$ é possível ainda observar que na maioria dos casos, com a aumento da vazão, o tempo de ruptura também diminui. Isso porque, segundo Geankoplis $(1993)^{34}$, o aumento da vazão acarreta em alterações nas resistências difusionais, diminuindo o tempo de contato do adsorvente com o adsorvato. Além disso, observa-se que as curvas de ruptura não apresentam a mesma inclinação nos dois sistemas, indicando que o mecanismo de retenção nos diferentes adsorventes não é o mesmo. Discussões sobre esses comportamentos distintos entre o carvão ativado de osso de boi tratado e não tratado serão abordadas no próximo item.
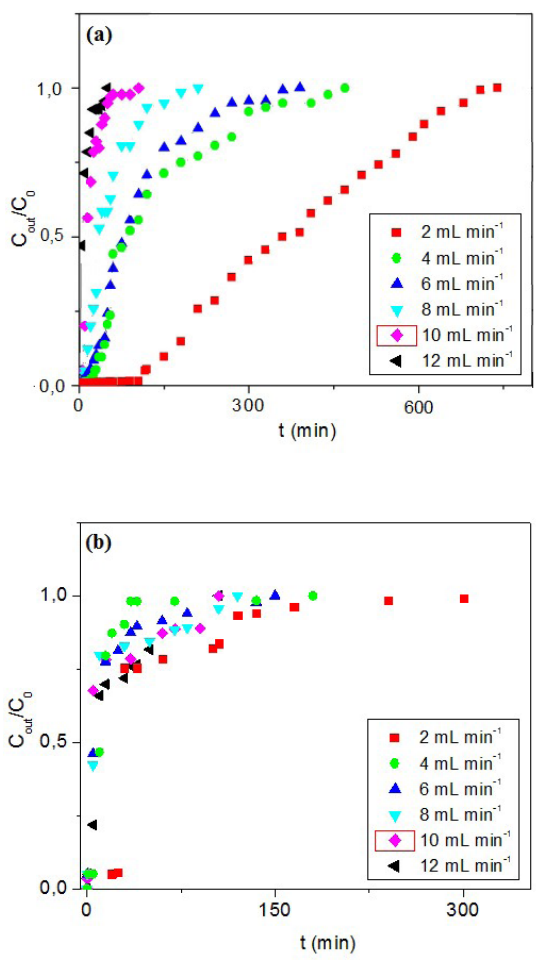

Figura 2. Curvas de ruptura para diferentes vazões (a) COB (b) COT. 
Na Tabela 1 são reportados os valores calculados para o comprimento de zona de transferência de massa (ZTM), razão operacional $\left(R_{0}\right)$, a porcentagem do leito ocupado $\left(Z T M / H_{t}\right)$ e capacidade dinâmica da coluna $\left(U_{i}^{t b}\right)$, em cada uma das vazões estudadas para os adsorventes em questão. Observa-se que para ambos a ZTM não ocupa a totalidade do leito de carvão em nenhuma das vazões estudadas. Para o $C O B$, a maior razão $Z T M / H_{t}$ foi obtida na vazão de $12 \mathrm{~mL}$

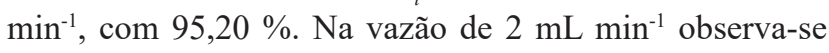
o menor comprimento de ZTM, indicando uma razão de 69,60 \%. Já para o COT, a vazão que levou a uma maior razão $Z T M / H_{t}$ foi a de $10 \mathrm{~mL}$ min-1, indicando que 98,08 \% do leito foram ocupados, contra $62,12 \%$ para a $2 \mathrm{~mL} \mathrm{~min}^{-1}$, vazão na qual também observa-se o menor comprimento da ZTM.

De acordo com Vijayaraghavan et al. (2005) $)^{35}$, o aumento da vazão resulta em redução da resistência à transferência de massa. A partir de um determinado ponto este efeito não é significativo e, portanto, existe uma vazão ótima de trabalho que pode ser determinada. Assim, o aumento da vazão propicia a diminuição do comprimento de ZTM até a vazão ótima e a partir daí, o comprimento da ZTM volta a aumentar. Acredita-se que, nestas condições, as resistências difusionais não são inversamente proporcionais ao aumento da vazão.

Segundo Song et al. $(2016)^{36}$, a capacidade de retenção até o ponto de ruptura pode diminuir com o aumento da vazão. Para vazões menores, a quantidade retida é maior, pois diminui-se a ZTM. Isto pode ser explicado pois, com o aumento da vazão, diminui-se o tempo de contato entre as moléculas de corante e os sítios de adsorção do carvão, o que diminui o tempo necessário para ocorrer a difusão.

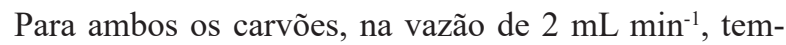
se o maior valor de $U_{i}^{\text {tb }}$, o que indica uma alta taxa de retenção de corante nesta vazão. À medida que se aumenta a vazão, há decréscimo desse parâmetro. Tal fato mostra que o corante azul $5 \mathrm{G}$ necessita de um tempo de contato relativamente grande para vencer os problemas estéricos decorrentes da difusão em adsorventes mesoporosos. Observa-se ainda que a quantidade retida de corante até o tempo de saturação é ligeiramente maior para o COT em todas as vazões, mesmo utilizando concentrações bem mais altas para o COT.

Além disso, valores de $R_{0}$ para a menor vazão indicam uma condição ideal de trabalho, ou seja, de mínima resistência difusional ${ }^{37}$. Assim, a vazão ótima de trabalho, isto é, a que minimiza os efeitos difusionais dos leitos foi a vazão de $2 \mathrm{~mL} \mathrm{~min}^{-1} \mathrm{em}$ ambos sistemas.

\section{Isoterma Dinâmica de Adsorção}

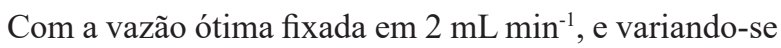
as concentrações, obteve-se curvas de ruptura em $30{ }^{\circ} \mathrm{C}$. Os resultados mostraram que o aumento da concentração proporciona uma diminuição no tempo de saturação do leito. Este fenômeno é comum para leitos fixos, uma vez que já

Tabela 1. Parâmetros de transferência de massa para a adsorção do corante Azul 5G em COB e COT associados às suas respectivas vazões volumétricas.

\begin{tabular}{|c|c|c|c|c|c|c|c|c|}
\hline \multirow{2}{*}{ 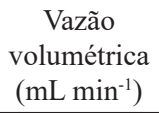 } & $\mathrm{COB}$ & COT & $\mathrm{COB}$ & COT & $\mathrm{COB}$ & COT & $\mathrm{COB}$ & $\mathrm{COT}$ \\
\hline & \multicolumn{2}{|c|}{ ZTM (cm) } & \multicolumn{2}{|c|}{$\mathrm{R} 0$} & \multicolumn{2}{|c|}{ Leito ocupado (\%) } & \multicolumn{2}{|c|}{ Uitb $\left(\mathrm{mg} \mathrm{g}^{-1}\right)$} \\
\hline 2 & 1,74 & 1,55 & 2,34 & 1,94 & 69,60 & 62,12 & 12,98 & 13,02 \\
\hline 4 & 1,97 & 2,31 & 4,06 & 13,42 & 78,80 & 92,48 & 6,24 & 7,36 \\
\hline 6 & 2,10 & 2,43 & 5,33 & 37,09 & 84,00 & 97,00 & 5,71 & 6,19 \\
\hline 8 & 2,00 & 2,42 & 4,30 & 35,45 & 80,00 & 96,96 & 4,70 & 5,34 \\
\hline 10 & 1,77 & 2,45 & 2,47 & 56,20 & 70,80 & 98,08 & 3,40 & 4,98 \\
\hline 12 & 2,38 & 2,37 & 3,44 & 18,82 & 95,20 & 94,80 & 0,31 & 2,33 \\
\hline
\end{tabular}


foi observado para diferentes adsorventes e adsorvatos ${ }^{38-40}$.

A Figura 3 apresenta os dados experimentais de equilíbrio correlacionados aos modelos de Langmuir e Freundlich para ambos os adsorventes. Segundo classificação de Giles et al. $(1960)^{41}$, a partir dos dados experimentais é possível observar um comportamento de isotermas do tipo L, subgrupo 1, no qual a quantidade adsorvida é proporcional à concentração da solução no início da curva. A curvatura inicial voltada para baixo, indica uma diminuição da disponibilidade dos sítios ativos com o aumento da concentração da solução.
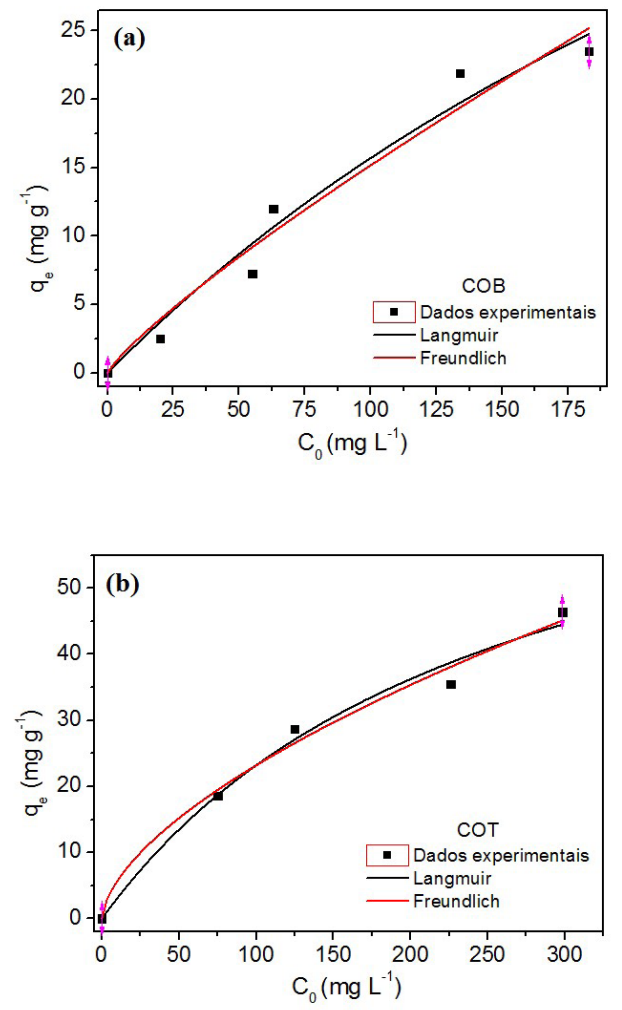

Figura 3. Isotermas de adsorção do corante Azul $5 \mathrm{G}$ ajustadas aos modelos de Langmuir e Freundlich para (a) COB (b) COT.
A análise dos parâmetros e coeficientes de correlação $\left(\mathrm{R}^{2}\right)$ apresentados na Tabela 2 para o COB e COT demonstram que tanto a isoterma de Langmuir quanto a de Freundlich representam satisfatoriamente os dados experimentais de equilíbrio, pois ambos os coeficientes são valores próximos à 1 . No entanto, o modelo de Langmuir apresentou valores de coeficiente de correlação relativamente maiores nos dois casos. Pelo parâmetro $R_{L}$ de Langmuir vê-se que adsorção do corante azul $5 \mathrm{G}$ em ambos os adsorventes é favorável $\left(0<R_{L}<1\right)$. Adicionalmente, o parâmetro $1<\mathrm{n}<10$ de Freundlich, também aponta que o processo de adsorção para o corante azul $5 \mathrm{G}$ é favorável ${ }^{42}$.

O melhor ajuste ao modelo da isoterma de Langmuir sugere que a adsorção do corante azul 5G nos adsorventes estudados dá-se pela formação de uma monocamada. Isto indica que têm-se um número fixo de sítios acessíveis disponíveis na superfície adsorvente, sendo que todos os sítios possuem a mesma energia. Dessa forma, cada sítio pode reter uma única molécula do corante $^{43}$. O modelo considera que as forças que atuam na adsorção são similares, em sua natureza, àquelas que envolvem combinação química, promovendo assim interações mais fortes entre adsorvente e adsorvato.

Tabela 2. Parâmetros das isotermas de adsorção.

\begin{tabular}{|c|c|c|c|}
\hline \multirow{2}{*}{ Modelo } & \multirow{2}{*}{ Parâmetros } & \multicolumn{2}{|c|}{ Adsorvente } \\
\hline & & $\mathrm{COB}$ & $\mathrm{COT}$ \\
\hline \multirow{4}{*}{ Langmuir } & $\begin{array}{c}\mathrm{qmax}(\mathrm{mg} \\
\mathrm{g}-1)\end{array}$ & 81,25 & 82,83 \\
\hline & KL (L mg-1) & 0,0024 & 0,0039 \\
\hline & RL & 0,7567 & 0,6724 \\
\hline & $\mathrm{R}^{2}$ & 0,9627 & 0,9849 \\
\hline \multirow{3}{*}{ Freundlich } & $\begin{array}{l}\mathrm{KF}((\mathrm{mg} \mathrm{g}-1) \\
(\mathrm{L} \mathrm{mg}-1) 1 / \mathrm{n})\end{array}$ & 0,3179 & 1,4085 \\
\hline & $\begin{array}{l}\mathrm{n}(\mathrm{g} \text { mg-1 } \\
\mathrm{min}-1)\end{array}$ & 1,19 & 1,64 \\
\hline & $\mathrm{R}^{2}$ & 0,9538 & 0,9815 \\
\hline
\end{tabular}


Outro parâmetro importante é o $\mathrm{q}_{\max }$ do modelo de Langmuir, que indica a capacidade máxima de adsorção do contaminante pelo adsorvente e pode ser utilizado para comparar sua eficiência ${ }^{44}$. É interessante avaliar, pela análise deste parâmetro, que houve um pequeno aumento na eficiência do processo de adsorção, utilizando-se o carvão ativado de osso de boi tratado com solução de hidróxido de sódio $\left(\mathrm{q}_{\max } 82,83 \mathrm{mg} \mathrm{g}-1\right)$, em detrimento do que é feito sem o tratamento $\left(\mathrm{q}_{\max } 81,23 \mathrm{mg} \mathrm{g}-1\right)$.

Segundo Jain e Gogate $(2017)^{45}$, o tratamento com $\mathrm{NaOH}$ aumenta a capacidade adsortiva do corante devido ao aumento das atrações eletrostáticas entre o adsorvente e adsorvato. Sabe-se que o corante azul $5 \mathrm{G}$ possui caráter aniônico e de acordo com os autores, o tratamento básico incorpora grupos cationicos $\left(\mathrm{Na}^{+}\right)$, fazendo com que o corante seja adsorvido seletivamente na superfície do COT. No entanto, observou-se neste estudo, que o tratamento básico não afetou significativamente a quantidade retida de corante. Acredita-se que a diminuição da área superficial específica após o tratamento $\left(123,3 \mathrm{~m}^{2} / \mathrm{g} \text { para } 83,6 \mathrm{~m}^{2} / \mathrm{g}\right)^{29}$ e a consequente diminuição de poros nesse caso, afetou o processo de adsorção mais do que o aumento de grupos básicos. Isso fez com que a retenção do corante não fosse tão eficiente como o esperado. Logo, acredita-se que a quimissorção tenha se sobressaído a fisiossorção no caso de adsorção do corante azul $5 \mathrm{G}$ em leitos fluidizados. Ou seja, a ocupação dos sítios foi mais significativa no processo de adsorção em relação as possíveis ligações em multicamadas dos grupos alcalinos com os do corante estudado.

A capacidade máxima de adsorção do carvão de osso de boi foi também comparada com a obtida por outros adsorventes, para o mesmo corante. Jesus et al. $(2011)^{46}$, obtiveram um valor de qmax de $43,38 \mathrm{mg} \mathrm{g}^{-1}$, utilizando bagaço de laranja seco como biossorvente. Já os pesquisadores Honório et al. (2015)47 conseguiram $72,43 \mathrm{mg} \mathrm{g}^{-1}$ de qmax utilizando resíduo de casca de soja. Observa-se assim, que o valor de $81,25 \mathrm{mg} \mathrm{g}^{-1}$ obtido para o COB neste trabalho, estabelece o uso deste adsorvente como promissor na remoção do corante azul 5G. O bom resultado obtido com o carvão ativado de osso pode ser justificado pela grande área superficial e pelo tamanho dos poros, que proporcionam um maior número de sítios de adsorção disponíveis para uma maior remoção de moléculas de corante ${ }^{43}$.

\section{Conclusão}

No presente estudo, o carvão ativado de osso de boi, em sua forma bruta e funcionalizada com $\mathrm{NaOH}$, foi avaliado na adsorção do corante reativo Azul 5G, em sistemas de leito fixo. Os estudos em coluna foram realizados variando a vazão de alimentação. Foi observado que a eficiência da coluna diminuiu com o aumento da vazão, sendo $2 \mathrm{~mL} \mathrm{~min}^{-1}$ a vazão ótima de trabalho encontrada para ambos os adsorventes. Os dados de equilíbrio foram melhor ajustados ao modelo de Langmuir, indicando a ocorrência de uma adsorção em monocamada. A capacidade máxima de adsorção para o COB e COT foi de 81,25 e $82,83 \mathrm{mg} \mathrm{g}^{-1}$, respectivamente. O leve aumento de qmax obtido com o tratamento básico realizado no adsorvente bruto revela que o processo de funcionalização da superfície química, com objetivo de melhorar o processo de adsorção do corante, não é viável. Ademais, o carvão ativado de osso de boi, um resíduo do processo de branqueamento do açúcar, demonstrou ser um adsorvente alternativo atraente e com potencial para ser usado na remoção do corante têxtil estudado.

\section{Agradecimentos}

Os pesquisadores são gratos à CAPES pelo auxílio financeiro.

\section{Referências}

1. ABIT. Perfil do setor. Associação Brasileira da Indústria Têxtil e Confecção. Disponível em: <http://www.abit.org.br/cont/perfil-dosetor>. Publicado em 2016. Accesso em: 8 out. 2017.

2. Taylor, P.; Manenti, D.R.; Trigueros, D.E.G.; Oliveira, A.P.; Borba, C.E, Kroumov, A.D. Assessment of the banana pseudostem as lowcost biosorbent for removal of the reactive blue 5G dye. Environ Technol. p. 37-41, 2015.

3. Kunz, A.; Peralta-Zamora, P.; Moraes, S.G. de; Durán, N. Novas tendências no tratamento de efluentes têxteis. Quim Nova, v. 25, n. 1, p. 78-82, 2002. 
4. Huang, Z.; Li, Y.; Chen, W.; Shi, J.; Zhang, N.; Wang, X.; Li, Z.; Gao, L.; Zhang, Y. Modified bentonite adsorption of organic pollutants of dye wastewater. Mater Chem Phys. v. 202, p. 266-276, 2017.

5. Mittersteiner, M.; Schmitz, F.; Barcellos, I.O. Reuse of dye-colored water post-treated with industrial waste: Its adsorption kinetics and evaluation of method efficiency in cotton fabric dyeing. J Water Process Eng. v.17, p. 181-187, 2017.

6. Marrakchi, F.; Khanday, W.A.; Asif, M.; Hameed, B.H. Cross-linked chitosan/sepiolite composite for the adsorption of methylene blue and reactive orange 16. Int J Biol Macromol. v. 93, p. 1231-1239, 2016.

7. Cheng, Z.; Zhang, L.; Guo, X.; Jiang, X.; Liu, R. Removal of Lissamine rhodamine $\mathrm{B}$ and acid orange 10 from aqueous solution using activated carbon/surfactant: Process optimization, kinetics and equilibrium. J Taiwan Inst Chem Eng. v. 47, p. 149-159, 2015.

8. Ruthven, D.M. Principles of Adsorption and Adsorption Process. New York: John Wiley \& Sons; 1984.

9. Goswami, M.; Phukan, P. Enhanced adsorption of cationic dyes using sulfonic acid modified activated carbon. J Environ Chem Eng. v. 5, n. 4, p. 3508-3517, 2017

10. Stawiński, W.; Węgrzyn, A.; Dańko, T.; Freitas, O.; Figueiredo, S.; Chmielarz, L. Acid-base treated vermiculite as high performance adsorbent: Insights into the mechanism of cationic dyes adsorption, regeneration, recyclability and stability studies. Chemosphere. v. 173, p. 107-115, 2017.

11. Sana, D.; Jalila, S. A comparative study of adsorption and regeneration with different agricultural wastes as adsorbents for the removal of methylene blue from aqueous solution. Chinese J Chem Eng. v. 25, n. 9, p. 1282-1287, 2017.

12. Gupta, V.K.; Agarwal, S.; Olgun, A.; Demir, H.I.; Yola, M.L.; Atar, $\mathrm{N}$. Adsorptive properties of molasses modified boron enrichment waste based nanoclay for removal of basic dyes. J Ind Eng Chem.v. 34, p. 244-249, 2016.

13. Kyzas, G.Z.; Lazaridis, N.K.; Kostoglou, M. Adsorption/desorption of a dye by a chitosan derivative: Experiments and phenomenological modeling. Chem Eng J. v. 248, p. 327-336,2014.

14. Gong, J.L.; Zhang, Y.L.; Jiang, Y.; Zeng, G.M.; Cui, Z.H.; Liu, K.; Deng, C.H.; Niu, Q.Y.; Deng, J.H.; Huan, S.Y. Continuous adsorption of $\mathrm{Pb}(\mathrm{II})$ and methylene blue by engineered graphite oxide coated sand in fixed-bed column. Appl Surf Sci. v. 330, p. 148-157, 2015

15. Mohammed, N.; Grishkewich, N.; Waeijen, H.A.; Berry, R.M.; Tam, K.C. Continuous flow adsorption of methylene blue by cellulose nanocrystal-alginate hydrogel beads in fixed bed columns. Carbohydr Polym. v. 136, p. 1194-1202, 2016.

16. Elmoubarki, R., Mahjoubi, F.Z.; Tounsadi, H.; Moustadraf, J.; Abdennouri, M.; Zouhri, A.; El Albani, A.; Barka, N. Adsorption of textile dyes on raw and decanted Moroccan clays: Kinetics, equilibrium and thermodynamics. Water Resour Ind. v. 9, p. 16-29, 2015.

17. Mendoza-Castillo, D.I.; Bonilla-Petriciolet, A.; Jáuregui-Rincón, J. On the importance of surface chemistry and composition of Bone char for the sorption of heavy metals from aqueous solution. Desalin Water Treat. v. 54, n. 6, p. 1651-1662, 2015.

18. Medellin-Castillo, N.A.; Leyva-Ramos, R.; Padilla-Ortega, E.; Perez, R.O.; Flores-Cano, J.V.; Berber-Mendoza, M.S. Adsorption capacity of bone char for removing fluoride from water solution. Role of hydroxyapatite content, adsorption mechanism and competing anions. J Ind Eng Chem. v.20, n. 6, p. 4014-4021, 2014.

19. Rojas-Mayorga, C.K.; Bonilla-Petriciolet, A.; Aguayo-Villarreal, I.A.; Hernández-Montoya, V.; Moreno-Virgem, M.R.; Tovar-Gómez, R.; Montes-Morán, R. Optimization of pyrolysis conditions and adsorption properties of bone char for fluoride removal from water. $\mathrm{J}$ Anal Appl Pyrolysis. v. 104, p.10-18, 2013.

20. Ko, D.C.K.; Cheung, C.W.; Choy, K.K.H.; Porter, J.F.; McKay, G. Sorption equilibria of metal ions on bone char. Chemosphere. v.54, n. 3, p. 273-281, 2004.

21. Chen, Y.N.; Chai, L.Y.; Shu, Y. Study of arsenic(V) adsorption on bone char from aqueous solution. J Hazard Mater. v. 160, n. 1, p. 168-172, 2008.

22. Pan, X.; Wang, J.; Zhang, D. Sorption of cobalt to bone char: Kinetics, competitive sorption and mechanism. Desalination. v. 249, n. 2, p. 609-614, 2009.

23. Reynel-Avila, H.E.; Mendoza-Castillo, D.I.; Bonilla-Petriciolet, A.; Silvestre-Albero, J. Assessment of naproxen adsorption on bone char in aqueous solutions using batch and fixed-bed processes. J Mol Liq. v.209, n.187-195, 2015.

24. Ip, A.W.M.; Barford, J.P.; McKay, G. A comparative study on the kinetics and mechanisms of removal of Reactive Black 5 by adsorption onto activated carbons and bone char. Chem Eng J. v. 157, n. 2-3, p. 434-442, 2010.

25. Ip, A.W.M.; Barford, J.P.; McKay, G. Reactive Black dye adsorption/ desorption onto different adsorbents: Effect of salt, surface chemistry, pore size and surface area. J Colloid Interface Sci. v. 337, n. 1, p. 32$38,2009$.

26. Walker, G.M.; Weatherley, L.R. Adsorption of dyes from aqueous solution - The effect of adsorbent pore size distribution and dye aggregation. Chem Eng J. v. 83, n. 3, p. 201-206, 2001.

27. Ghanizadeh, G.; Asgari, G. Adsorption kinetics and isotherm of methylene blue and its removal from aqueous solution using bone charcoal. React Kinet Mech Catal. v. 102, n. 1, p. 127-142, 2011.

28. Silva, P.H.R.; Barbosa, J.G.; Barella, R.A.; Araújo, A.B.C.; Ostroski, I.C. Comparative study of the adsorption of cadmium and zinc on 
activated bone char. Acta Sci Technol. v.39, n. 2, p. 215, 2017.

29. Winter, C.; Caetano, J.N.; Araújo, A.B.C.; Chaves, A.R.; Ostroski, I.C.; Vaz, B.G.; Perez, C.N. Activated carbons for chalcone production: Claisen-Schmidt condensation reaction. Chem Eng J. v. 303, p. 604-610, 2016.

30. Geankoplis, C.J. Transport Processes and Unit Operations. 4th ed. USA; 2003.

31. McCabe, W.L.; Smith, J.C. Unit Operations of Chemical Engineering. 6th ed. McGraw-Hill International; 2005.

32. Pereira, M.R.; Arroyo, P.A.; Barros, M.A.S.D.; Sanches, V.M.; da Silva, E.A.; Fonseca, I. M.; Lovera, R.G. Chromium adsorption in olive stone activated carbon. Adsorption. v. 12, n. 2, p. 155-162, 2006.

33. Gazola, F.C.; Pereira, M.R.; Barros, M.A.; Silva, E.; Arroyo, P. Removal of $\mathrm{Cr} 3+$ in fixed bed using zeolite NaY. Chem Eng J. v. 117, p. 253-261, 2006.

34. Geankoplis, C.J. Transport Processes and Unit Operations. 3rd ed. PTR Prentice Hall; 1993.

35. Vijayaraghavan, K.; Jegan, J.; Palanivelu, K.; Velan, M. Biosorption of copper, cobalt and nickel by marine green alga Ulva reticulata in a packed column. Chemosphere. v. 60, p. 419-426, 2005.

36. Song, S.; Hau, Y.; Saman, N.; Johari, K.; Cheu, S-C.; Kong, H.; Mat, $\mathrm{H}$. Process analysis of mercury adsorption onto chemically modified rice straw in a fixed-bed adsorber. J Environ Chem Eng. v. 4, n. 2, p. 1685-1697, 2016.

37. Netpradit, S.; Thiravetyan, P.; Towprayoon, S. Evaluation of metal hydroxide sludge for reactive dye adsorption in a fixed-bed column system. Water Res. v. 38, p. 71-78, 2004.

38. Lakshmipathy, R.; Sarada, N.C. A fixed bed column study for the removal of $\mathrm{Pb} 2+$ ions by watermelon rind. Environ Sci Water Res Technol. v. 1, n. 2, p. 244-250, 2015.

39. Tamez Uddin, M.; Rukanuzzaman, M.; Maksudur Rahman Khan, M.; Akhtarul Islam, M. Adsorption of methylene blue from aqueous solution by jackfruit (Artocarpus heteropyllus) leaf powder: A fixedbed column study. J Environ Manage. v. 90, n. 11, p. 3443-3450, 2009.

40. Purkait, M.K.; DasGupta, S.; De, S. Adsorption of eosin dye on activated carbon and its surfactant based desorption. J Environ Manage. v. 76, n. 2, p. 135-142, 2005.

41. Giles, C. H.; MacEwan, T. H.; Nakhwa, S.N.; Smith, D. Studies in adsorption. Part XI. A system of classification of solution adsorption isotherms, and its use in diagnosis of adsorption mechanisms and in measurement of specific surface areas of solids. J Chem Soc. v. 846, n. 3973-3993, 1960.

42. Chandra, V.; Park, J.; Chun, Y.; Lee, J.W.; Hwang, I-C.; Kim, K.S.
Water-Dispersible Magnetite-Reduced Graphene Oxide Composites for Arsenic Removal. ACS Nano. v. 4, n. 7 p. 3979-3986, 2010.

43. Sewu, D.D.; Boakye, P.; Woo, S.H. Highly efficient adsorption of cationic dye by biochar produced with Korean cabbage waste. Bioresour Technol. v. 224, p. 206-213, 2017.

44. Hu, Z.P.; Gao, Z.M.; Liu, X.; Yuan, Z.Y. High-surface-area activated red mud for efficient removal of methylene blue from wastewater. Adsorpt Sci Technol. v. 0, p. 1-18, 2017.

45. Jain, S.N.; Gogate, P.R. Acid Blue 113 removal from aqueous solution using novel biosorbent based on $\mathrm{NaOH}$ treated and surfactant modified fallen leaves of Prunus Dulcis. J Environ Chem Eng. v. 5, n. 4, p. 3384-3394, 2017.

46. Jesus, A.M.D.; Romão, L.P.C.; Araújo, B.R.; Costa, A.S.; Marques, J.J. Use of humin as an alternative material for adsorption/desorption of reactive dyes. Desalination. v. 274, n. 1, p. 13-21, 2011.

47. Honorio, J.F.; Veit, M.T.; Cunha-Gonçalves, G.; Campos, E.A.; Fagundes-Klen, M.R. Adsorption of reactive blue BF-5G dye by soybean hulls: Kinetics, equilibrium and influencing factors. Water Sci Technol. v.73, n. 5, p. 1166-1174, 2016.

\section{Noemi H. de Melo, Maria E. O. Ferreira, Nathan F. F. Potenciano, Paulo R. Martins \& Indianara C. Ostroski*}

Instituto de Química, Universidade Federal de Goiás, CEP 74690-900 Goiânia, Goiás, Brasil.

*E-mail: indianara_ostroski@ufg.br 